\title{
Scanning Helium Ion Microscopy-Induced Secondary Electron Yields of Composite Materials
}

\author{
Vighter Iberi $^{1,2}$, Uk Huh ${ }^{2}$, Yueying Wu ${ }^{2}$, Philip D. Rack ${ }^{1,2}$, Adam J. Rondinone ${ }^{1}$, David C. Joy ${ }^{1,2}$ \\ ${ }^{1 .}$ Center for Nanophase Materials Science, Oak Ridge National Laboratory, Oak Ridge, TN 37831, USA \\ 2. Department of Materials Science and Engineering, University of Tennessee, Knoxville, TN 37996, \\ USA
}

This project attempts to quantify the ion-generated secondary electron (iSE) yields of pure elements and composite materials generated by scanning helium ion microscopy. Additionally, the effects of surface roughness and purity on the experimental iSE yields of materials are investigated.[1] Secondary electron imaging has been the most robust method of obtaining high-resolution images and is at the core of imaging and signal analysis in scanning electron and ion microscopy.

Briefly, stainless steel, copper and silicon samples were obtained and used without further purification. Gold ( $\mathrm{Au}$ ) and silver (Ag) were co-sputtered using radio frequency (RF) magnetron sources to form a $\sim 200 \mathrm{~nm}$ Au-Ag alloy film with a gold composition ranging from 25-75 at\% along the diameter of a $100 \mathrm{~mm}$ silicon (100) wafer. The measured sputtering rates at the center of the substrate was $3 \mathrm{~nm} / \mathrm{min}$ for both gold and silver under the following conditions $-5 \mathrm{~cm}$ diameter sputter targets and a RF power of $20 \mathrm{~W}$ for both targets, and a processing pressure of $25 \mathrm{sccm}$ Ar at 5 mTorr.

Scanning helium ion microscopy was performed using a Carl Zeiss ORION Nanofab microscope, operating at a fixed extraction voltage of $30 \mathrm{kV}$ while the accelerating voltages were set to $15 \mathrm{kV}, 25 \mathrm{kV}$ and $29 \mathrm{kV}$ for each sample. The helium gas pressure in the column was maintained at $2 \times 10^{-6}$ Torr and a probe current of $\sim 1.3-1.6 \mathrm{pA}$, measured from the beam blanker, was achieved using a $20 \mu$ aperture and a spot control of 7. Secondary electrons ejected from each material were collected using an Everhart-Thornley (ET) detector without applying any bias to the sample. This was done in order to avoid the introduction of electric fields that may affect the landing energy of the ion beam. Other possible effects occurring within the specimen chamber were taken into account by using silicon as a reference material throughout the experiment. The experimental iSE yield of each material was obtained by performing a histogram analysis routine in ImageJ (available as public domain software from http://rsb.info.nih.gov/ij/download.html) on each image.

References:

[1] D. C. Joy in "Helium Ion Microscopy, Principles and Applications”, Springer, (New York) p. 27. [2] Scanning Helium ion experiments were conducted at the Center for Nanophase Materials Sciences, Oak Ridge National Laboratory, which is a DOE Office of Science User Facility. Gold and silver composite materials were prepared at the Department of Materials Science and Engineering, University of Tennessee Knoxville. 
